\title{
Intervención logopédica sobre la comprensión lectora en una adolescente con síndrome de Down
}

\author{
Samuel Sacido López \\ Universidade da Coruña
}

\begin{abstract}
Resumen
El objetivo principal del presente fue mejorar la comprensión lectora en una adolescente con síndrome de Down. El trabajo se llevó a cabo a lo largo de tres fases: una evaluación previa a la intervención, una intervención centrada en la comprensión lectora; y una evaluación al finalizar el período de tratamiento. Los resultados muestran una mejoría en las subpruebas de estructuras gramaticales y de comprensión de textos, así como en signos de puntuación y velocidad lectora, medidos a través del PROLEC-R.
\end{abstract}

\section{Introducción}

Los sujetos con síndrome de Down presentan diferentes dificultades asociadas al síndrome de Down que podrían clasificarse en alteraciones del sistema nervioso, del desarrollo psicomotor, del desarrollo socioafectivo, del desarrollo del lenguaje, y del desarrollo cognitivo (Arnaiz y Martínez, 2002).

Con respecto a las alteraciones del sistema nervioso, producen ciertas dificultades relacionadas con la atención, con la memoria a corto plazo y procesamiento de la información, con la memoria a largo plazo, y por último, con el análisis (Arnaiz y Martinez, 2002).

En cuanto a las características del desarrollo psicomotor, las personas con síndrome de Down presentan una falta de psicomotricidad generalizada, además de la hipotonía muscular e hiperflexibilidad articular, marcha atípica, problemas en el equilibrio, y en la capacidad para el control postural. Además presentan un tiempo de reacción dos veces más largo que los sujetos que no tienen el trastorno, por lo que la rapidez motora está por debajo de la edad cronológica real, como mencionan Anson y Davis en Arnaiz y Martinez, 2002.

Los niños con síndrome de Down desarrollan sus habilidades sociales más lentamente que sus iguales en edad cronológica pero que no presentan el trastorno. Siendo así, la sonrisa social aparece 3 semanas más tardía a la de los sujetos sin síndrome de Down. Además no presentan eficacia a la hora de realizar la interacción con su ambiente social.

En cuanto al desarrollo del lenguaje, es el área que presenta un mayor retraso en los niños con síndrome de Down, especialmente en el aspecto expresivo. Siendo frecuentes las dificultades en el desarrollo fonológico, semántico, morfosintáctico y pragmático (Arnaiz y Martinez, 2002).

Otra de las características que presentan los individuos con síndrome de Down, como señalan Arnaiz y Martínez
(2002), es un cuadro importante de "retraso mental", concepto que se sustituyó en el actual DSM-5 (American Psychiatric Association, 2014), por el término "discapacidad intelectual" o lo que es lo mismo, "trastorno del desarrollo intelectual".

Esta discapacidad intelectual produce, a su vez, una serie de dificultades de aprendizaje: aprendizaje del cálculo, aprendizaje de la escritura, aprendizaje y desarrollo de la lectura fluida y comprensiva, y la imposibilidad de aplicar las reglas ortográficas. Con respecto a las dificultades lectoras, la investigación muestra que los niños con síndrome de Down presentan más problemas de tipo comprensivo que de fluidez lectora. La comprensión lectora es una cualidad que se puede definir como elemento intrínsecamente constitutivo de la actividad lectora, ya que el objetivo básico de la lectura es enterarse y conocer el contenido de lo que se lee (Troncoso y Flórez, 2011).

Para llevar a cabo una correcta y eficaz lectura, intervienen una serie de procesos cognitivos como el proceso perceptivo, léxico, sintáctico y semántico (González y Villalba, 2007).

El presente trabajo se centra en una intervención para mejorar la comprensión lectora en una adolescente con síndrome de Down, por eso señalar que la capacidad para descifrar lo leído y la capacidad para comprenderlo se desarrollan de manera independiente, yendo la primera por delante de la segunda. A las personas con síndrome de Down les resulta más fácil leer, y con mejores resultados, que comprender lo que se lee y lo que se oye (Troncoso y Flórez, 2011).

Los autores arriba mencionados llevaron a cabo un estudio que puso de manifiesto que la habilidad para descifrar lo leído superaba a la capacidad para comprenderlo, además de determinar también que la comprensión lectora estaba por encima de la capacidad de escucha. Por esto, se fijó que la capacidad para comprender lo leído guardaba relación con la capacidad para comprender lo escuchado. Asimismo, constataron que las habilidades para leer son independientes de las habilidades tanto para la comprensión de lo leído como de lo escuchado, y que la comprensión lectora viene determinada directamente por la comprensión de lo escuchado.

Por otro lado, también se defiende la idea de que la comprensión lectora es un producto de la interacción entre lector y lectura, lo que implica que la memoria a largo plazo juega un papel importante en la comprensión lectora (Johnston, 1989). Sin embargo, otros autores afirman que la comprensión es un proceso que tiene lugar 
en cuanto se recibe la información y en el que sólo trabaja la memoria inmediata (Carroll, 1971). Royer y Cunningham (1978), afirman que los procesos de comprensión y los procesos de la memoria están muy unidos.

De estas tres posturas teóricas, la que se considera más acertada es la defendida por Royer y Cunningham (1978), puesto que para la comprensión lectora, además de los procesos implicados en la lectura, citados anteriormente y entre los que se encuentran los procesos perceptivo, léxico, sintáctico y semántico, también se une a una eficaz comprensión de la lectura la capacidad para comprender lo escuchado, que va directamente relacionado con la comprensión lectora, de la misma manera que se encuentra vinculado a un necesario almacenamiento de la información tanto en la memoria inmediata como en la memoria a largo plazo, ya que un mensaje comprendido, será retenido en la memoria mejor que uno no comprendido.

Pero el área de debilidad de la población con síndrome de Down es la memoria a corto plazo, sobre todo en tareas en las que cuanto más nivel de carga de procesamiento se precise, más pobre es el recuerdo de la información verbal recibida, porque aunque la carga de procesamiento sea poca, mostraran malos resultados, pero mucho peores si la carga es mayor (Roch, Florit y Levorato, 2012). Esta afirmación viene dada por un estudio de Roch, Florit y Levorato (2011), en el que los resultados mostraron que la memoria verbal contribuye de una manera mucho más alta a la comprensión de la lectura de lo que lo hacen las habilidades lingüísticas. Sin embargo, hay que tener en cuenta que si la memoria verbal es sobrecargada procesando demandas, será difícil integrar la información expresada en el texto y construir la representación semántica, por lo que hay un proceso de compromiso entre las demandas del proceso de comprensión y la cantidad de información que se puede almacenar (Daneman y Carpenter, 1983).

Todo este compendio de procesos citados anteriormente, son necesarios para que una persona pueda comprender lo que lee, pero en el caso de una persona con síndrome de Down esto no es así, pues como he citado anteriormente, la comprensión lectora se ve gravemente afectada, ya que va desfasada con respecto a la comprensión de lo escuchado y la memoria verbal a corto plazo.

El presente trabajo describe un estudio de caso llevado a cabo con una adolescente con síndrome de Down. El objetivo principal del mismo fue mejorar la comprensión lectora de esta adolescente. El trabajo se diseñó a lo largo de 3 fases: una primera fase de evaluación en la que se recogieron datos acerca de la lectura y las habilidades narrativas; una segunda fase en la que se puso en práctica a lo largo de un mes una intervención logopédica en el contexto escolar para mejorar la comprensión lectora; y finalmente una evaluación realizada al final del período de tratamiento para comprobar si se habían producido cambios en las variables de interés. A continuación se describe el desarrollo del estudio, se exponen los resultados obtenidos, y se analiza la importancia de llevar a cabo intervenciones de este tipo.

\section{Método}

\section{Participantes}

En este estudio participó una niña de 17 años y 11 meses de edad, de aquí en adelante $\mathrm{G}$, diagnosticada de síndrome de Down, que acudía al colegio concertado Compañía de María en la ciudad de A Coruña. Aunque cursa $4^{\circ}$ de Educación Secundaria Obligatoria, necesita una adaptación curricular de $5^{\circ}$ de Educación Primaria, por lo que asistía al aula de Educación Especial para trabajar las distintas materias de su adaptación curricular.

\section{Instrumentos}

La evaluación se llevó a cabo a lo largo de dos fases: la primera correspondió con la evaluación previa a la intervención, y la segunda con la evaluación posterior a la intervención. En ambas fases, se utilizaron dos instrumentos: la batería de Procesos Lectores Revisada (PROLEC-R) (Arribas, Cuetos, Rodríguez y Ruano, 2007), y la prueba de Retelling (Stein y Glenn, 1979). A continuación se describen cada uno de los instrumentos.

La Batería de Procesos Lectores Revisada (PROLEC-R) (Arribas et al., 2007) es una prueba estandarizada que tiene como objetivo evaluar los procesos lectores mediante cuatro bloques principales y tres escalas de índices.

La prueba de Retelling (Stein y Glenn, 1979). Se trata de una prueba que evalúa la narración y la comprensión. La narración se evalúa mediante un texto traducido al español con el título de "Atrapado vivo", formado por 5 episodios, que a su vez están formados cada uno por seis componentes, y con un total de 47 secuencias.

En cuanto a la fase de intervención se emplearon distintos materiales en función de las tareas planteadas. Esto es, se utilizó un ordenador para llevar a cabo diferentes tareas como el visionado de algún video o para realizar actividades que requieren un uso informático debido a que están accesibles en páginas web. Por otra parte, también se elaboraron fichas con diferentes imágenes en las que la niña debería asociar la imagen con otro símbolo que le correspondiera.

\section{Procedimiento}

Este trabajo se realizó durante un mes y medio. Una vez seleccionado el caso a intervenir, se realizó una evaluación previa a la intervención que constó de tres sesiones de 50 minutos cada una. En la evaluación se le administraron, en primer lugar, el PROLEC-R, siendo necesarias dos sesiones de 50 minutos para su completa administración, y en segundo lugar, y ya en la tercera sesión, se aplicó la prueba de Retelling, que ocupó un espacio en el tiempo de 20 minutos.

En función de los resultados obtenidos tras la fase de evaluación, se diseñó un plan de intervención con tres objetivos principales sobre los que se iban a desarrollar las actividades: comprensión lectora, comprensión oral y atención y concentración.

En cuanto a la fase de intervención, se comenzó el proceso una vez terminada la fase de evaluación, y tras el diseño de actividades para trabajar los objetivos planteados en la programación. A continuación, en la 
tabla 1, se recogen los objetivos planteados para la intervención con la adolescente.

Tabla 1

Objetivos planteados para la intervención

\section{Comprensión lectora:}

Potenciar la comprensión lectora dentro del aula escolar

Despertar y aumentar el interés de la alumna por la lectura.

Descubrir la lectura como fuente de información del mundo que rodea a la alumna.

\section{Comprensión oral:}

Aumentar la comprensión oral.

Atención y concentración:

Mejorar la atención y la concentración dentro del aula escolar.

\section{Resultados}

A continuación se describen los resultados obtenidos haciendo una comparación de los datos obtenidos en cada una de las pruebas empleadas tanto en la fase previa a la intervención como una vez finalizada la misma. En primer lugar se exponen los resultados obtenidos con el test de Procesos Lectores Revisado (PROLEC-R) (Arribas et al, 2007).

En la escala índices principales se pudo observar que $\mathrm{G}$ mejoró en la subprueba de estructuras gramaticales obteniendo en la fase pre-intervención una puntuación directa de 12 lo que indicaba (D), mientras que en la fase post-intervención obtuvo una puntuación de 14, que indica un nivel de normalidad. Así mismo, en la subprueba de comprensión de textos también se produce una mejoría al pasar de la puntuación de 7 en la fase previa a la intervención a la puntuación de 10 en la fase posterior, lo que indica que paso de la categoría (D) a la categoría $(\mathrm{N})$.

En la escala de índices de precisión la joven obtuvo en la subprueba de signos de puntuación una puntuación directa de 10, correspondiente a una categoría de normal en la evaluación posterior a la intervención, después de haber obtenido en la fase previa a la intervención una puntuación directa de 9 correspondiéndose con la categoría de duda..

Finalmente, en la escala índice de velocidad, la adolescente obtuvo las mismas categorías en las distintas subpruebas que en la evaluación previa a la intervención, pero no los mismos segundos, pues la subprueba de lectura de palabras la realizó en 45 segundos, la de pseudopalabras en 57 segundos y por último, la subprueba de signos de puntuación la realizó en 74 segundos.

Los resultados obtenidos con la prueba de Retelling (Stein y Glenn, 1979) se divide en dos grandes bloques: narración y comprensión. A su vez, el bloque de narración analiza seis componentes diferentes (planteamiento informativo, suceso inicial, respuesta interna, intento-acción, consecuencia directa y reacción) y el bloque de comprensión se divide en preguntas referidas a la comprensión de detalles e información general y comprensión de tipo inferencial.

En el bloque de narración, y haciendo una comparación entre los resultados de las evaluaciones previa y posterior a la intervención, se observa que $G$ presenta una longitud de narración similar tanto en la fase pre-intervención como en la fase post-intervención, ya que en la fase anterior a la intervención presenta un total de 12 secuencias mientras que en la evaluación posterior a la intervención presenta 10 . G es capaz de dar información de los seis tipos de secuencias, además incrementa el número de secuencias citadas en planteamiento informativo y suceso inicial, sin embargo hubo descreimiento en las citas de las secuencias intento-acción, consecuencia directa y reacción, mientras que se mantuvo en el bloque de las secuencias de reacción.

Es necesario señalar que aunque es capaz de narrar dando información de los 6 tipos de secuencias, tanto en la evaluación pre como en la post-intervención la puntuación obtenida por $\mathrm{G}$ en la mayoría de ellas era de 1 punto (ver tabla 6), lo que indicó que la narración de las mismas resultaba incompleta.

Por otra parte, la coherencia de la narración también fue mejor en la fase pre-intervención, pues presenta un total de 2 episodios, mientras que en la evaluación posterior a la intervención el número total de episodios es de sólo 1.

En cuanto al bloque de comprensión, se observó que G aumentó en un punto los resultados en cuanto a las preguntas de comprensión de detalles e información general, ya que de las ocho preguntas formuladas contesto correctamente a cinco. Mientras que se mantuvo con la misma puntuación en las preguntas de tipo inferencial, tanto en la evaluación previa como posterior a la intervención, alcanzando seis puntos de los ocho posibles, lo que indica que su nivel de comprensión ha mejorado.

Finalmente, los datos recogidos mediante la observación informal en las sesiones de intervención ponen de manifiesto que antes de la intervención, la joven no sostenía la atención todo el tiempo necesario para la realización de alguna tarea, siendo factores externos los culpables de las distracciones. A pesar de no focalizar un trabajo exhausto sobre este objetivo, y tras la observación de las últimas sesiones de la intervención, la joven se mostraba más atenta a la par que concentrada a la hora de realizar las tareas.

\section{Discusión y conclusión}

Como ya se dijo, los objetivos principales en este estudio fueron mejorar la comprensión lectora, la comprensión oral y la atención y concentración en una adolescente que presentaba síndrome de Down. La evaluación previa y posterior a la intervención mostró unos resultados en los cuales se observa una mejoría en la comprensión lectora. 
Los resultados obtenidos a través de la evaluación llevada a cabo con el PROLEC-R (Cuetos et al., 2007) en la fase posterior a la intervención reflejan mejoría con respecto a los resultados de la fase previa a la intervención. Concretamente, se observa mejoría en las subpruebas de estructuras gramaticales y de comprensión de textos dentro de la escala de índices principales, mientras que en la escala de índices de precisión se observa mejoría en la subprueba de signos de puntuación, al igual que en la escala de índices de velocidad en la que las tres subpruebas que la conforman G alcanza mejores resultados que en la evaluación anterior a la intervención. Sin embargo, no se refleja ningún retroceso en el resto de subpruebas que conforman el test y en las que la joven no obtiene mejores resultados en la fase post-intervención en comparación con la fase pre-intervención, tanto en la escala de índices principales, índices de precisión e índices de velocidad, no produciéndose cambios con respecto a la evaluación inicial, lo que puede deberse a que la intervención planteada no fuera la más óptima para trabajar los contenidos de dichas subpruebas, así como el corto tiempo de intervención llevado a cabo, ya que la joven podría necesitar que la intervención fuese más duradera en el tiempo. No obstante, los resultados en los que se observa mejoría, se produjeron después de haber realizado un programa de intervención orientado especialmente a la mejora de la comprensión lectora de $\mathrm{G}$, lo que coincide con los resultados obtenidos en trabajos como los de Roch et al. (2011) o Troncoso y Flórez (2011), en los que se pone de manifiesto que la comprensión lectora mejora tras un plan de intervención en que se tenga en cuenta la comprensión de lo escuchado, ya que es un elemento de predicción del nivel de comprensión de lo leído. Además, en algunas de las actividades se trabajó la comprensión de lo escuchado, pues coincidiendo también con el estudio de Troncoso y Flórez (2011), es un factor que influye directamente sobre la comprensión lectora.

Asimismo, como ya se ha mencionado al principio del anterior párrafo, se mostró mejoría en las subpruebas de estructuras gramaticales y signos de puntuación, lo que explica que gracias a la mejor comprensión lectora, G también incrementó su potencial de habilidad lectora.

En cuanto a los resultados obtenidos en la prueba de Retelling (Stein y Glenn, 1979), se observa un avance en la comprensión oral con respecto a la evaluación inicial, mientras que la parte de narración se encuentra más o menos similar con respecto a la fase pre-intervención.

En el bloque de comprensión, $\mathrm{G}$ alcanza 1 punto más en la evaluación post-intervención en las preguntas de comprensión de detalles e información general, llegando a obtener 5 puntos de los 8 posibles, mientras que en las preguntas de tipo inferencial se mantiene con 6 puntos de 8, es decir, igual que en la fase previa a la intervención. Por ello, se puede decir que a nivel general la joven obtiene una buena puntuación en cuanto a la comprensión oral, porque aunque la diferencia entre ambas fases de evaluación es de simplemente 1 punto en ambos tipos de preguntas, tiene un elevado número de puntos en cada tipo de preguntas.
En cuanto al bloque de narración, las puntuaciones de ambas fases de evaluación fueron similares. En la evaluación post-intervención, G fue capaz de conseguir elaborar 1 episodio completo formado por los componentes de respuesta interna, intento-acción y consecuencia directa, mientras que en la fase pre-intervención había sido capaz de formar 2 episodios completos de 5 posibles. Aún así, la coherencia de la narración es mejor que la longitud de la misma, pues la joven obtuvo en la longitud de narración un total de 10 secuencias de 47 posibles en la fase post-intervención, alcanzando 12 en la fase pre-intervención. Que los resultados se mantengan similares de una fase de evaluación a otra podría ser debido a varios factores. Como se citó anteriormente, se puede deber a que la intervención planificada no fuera la óptima para los contenidos de la narración, o que el proceso de intervención tuviese que ser más duradero. No obstante, en este caso y bajo mi criterio, estos resultados se pudieron deber a que el día anterior al que se realizó la sesión en la que se llevó a cabo la evaluación post-intervención la joven había celebrado su $18^{\circ}$ cumpleaños, por lo que en el momento de realizar la evaluación podía estar despistada debido a factores externos relacionados con vivencias del día anterior, pues aunque en el período de intervención no hubiese mejoría, no tendría por qué obtener menor puntuación que la alcanzada en la primera evaluación realizada.

Es importante decir que la atención y concentración fue una limitación de este estudio, pues no se evaluó de manera más exhaustiva, si no que se realizó una observación informal, lo que no facilitó la orientación de la intervención sobre esas dificultades precisas.

Por último, decir que el resultado global de la intervención fue positivo, teniendo en cuenta que la duración de la intervención fue de sólo un mes. Aunque G obtuvo resultados más positivos en la fase post-intervención, se considera necesario mantener la intervención durante el curso escolar, procurando trabajar todos los objetivos marcados en el programa de intervención y profundizando más en aquellas actividades con las que se trabajaba la atención y la comprensión lectora, para generalizar los aprendizajes y alcanzar un mayor nivel en estos.

Para finalizar, y a modo de conclusión general, se puede decir que existe una relación directa entre la comprensión lectora y una intervención programada para este aspecto, pues basándose en los resultados de este estudio de caso se observa la mejoría alcanzada en un corto periodo de tiempo. Sin embargo, las líneas de investigación en relación a la comprensión lectora en síndrome de Down deberían ampliarse a lo largo del tiempo para conocer profundamente las bases que sustenten una intervención correcta sobre esta variable.

\section{Referencias bibliográficas}

American Psychiatric Association (APA). (2014). Diagnostic and Statistical Manual of Mental Disorders, fifth edition DSM-V. Washington, DC: American Psychiatric Publishing. 
Arnaiz, P., y Martínez, R. (2002). Alumnos con discapacidad intelectual asociada a síndrome de Down: características del desarrollo y principios neuropsicopedagógicos de la respuesta educativa. En J.M. García, J. Pérez, y P.P. Berruezo (Eds), Discapacidad intelectual. Desarrollo, comunicación e intervención (pp. 349-369). Madrid: CEPE.

Carroll, J.B. (1971). Learning from verbal discourse in educational media. Princeton NJ: Educational Testing Service.

Daneman, M., y Carpenter, P. (1983). Individual differences in working memory and reading. Journal of Verbal Learning and Verbal Behavior, 19(4), 450-466.

González, P. (2007). Evaluación e intervención sobre las dificultades en la intervención lectora. En L. González, y A. Villalba (Eds.), Tratamiento educativo de los trastornos de la Audición y el Lenguaje (pp. 45-79). Madrid: Gento Palacios.

Johnston, P.H. (1989). La evaluación de la comprensión lectora. Madrid: Visor.
Roch, M. Florit, E., y Levorato, M. (2011). A follow-up study on reading comprehensionin Down syndrome: The role of reading skills and listening comprehension. International Journal of Languaje and Communication Disorders, 46(2), 231-242.

Roch, M., Florit, E., y Levorato, M. (2012). The advantage of reading over listening text comprehension in Down syndrome: What is the role of verbal memory? Research in Developmental Disabilities, 33(3), 890-899.

Royer, J., y Cunningham, D. (1978). Center for the Study of Reading. Cambridge: The National Institute of Education.

Stein, N. y Glenn, C. (1979). An analysis of story comprehension in elementary school children. En: R.O. Freedle (Ed.). Advances in discourse processing (vol. 2). Norwood, NJ: Ablex.

Troncoso, M.V., y Flórez, J. (2011). Comprensión en la lectura de las personas con síndrome de Down. Revista Síndrome de Down, 28(2), 50-59. 